\title{
Racial variation in cardiovascular morbidity and mortality in essential hypertension
}

\author{
R S Khattar, J D Swales, R Senior, A Lahiri
}

\begin{abstract}
Objectives-To perform a longitudinal comparison of morbidity and mortality among white, south Asian and Afro-Caribbean hypertensive patients in relation to baseline demographic characteristics and clinic and ambulatory blood pressure variables.

Design-Observational follow up study.

Setting-District general hospital and community setting in Harrow, England.

Patients-528 white, 106 south Asian, and 54 Afro-Caribbean subjects with essential hypertension who had undergone 24 hour ambulatory intra-arterial blood pressure monitoring. Interventions-Follow up for assessment of all cause morbidity and mortality over a mean (SD)
\end{abstract} of 9.2 (4.1) years.

Main outcome measures-Non-cardiovascular death, coronary death, cerebrovascular death, peripheral vascular death, non-fatal myocardial infarction, non-fatal stroke, coronary revascularisation.

Results-South Asians had the highest all cause event rate of 3.46, compared with 2.50 (NS) and $0.90(\mathrm{p}=0.002)$ events/100 patient-years for whites and Afro-Caribbeans, respectively. This was because of an excess of coronary events (2.86 $v 1.32$ events/100 patient-years in south Asians $v$ whites, respectively; $\mathrm{p}=0.002)$. Age $(\mathrm{p}<0.001)$, sex $(\mathrm{p}<0.001)$, race (south Asians : whites, hazard ratio $1.79 ; \mathrm{p}=0.008)$, diabetes $(\mathrm{p}=0.05)$, previous history of cardiovascular disease $(\mathrm{p}<0.001)$, and 24 hour ambulatory systolic blood pressure $(\mathrm{p}=0.006)$ were independent predictors of time to a first event. Clinic blood pressure did not provide additional prognostic information.

Conclusions-South Asian origin was an independent predictor of all cause events, mainly because of an excess of coronary events in this group. Ambulatory but not clinic blood pressure was of additional value in predicting subsequent morbidity and mortality.

(Heart 2000;83:267-271)

Keywords: race; hypertension; prognosis; ambulatory blood pressure

Hypertension appears to be more common in Afro-Caribbean and south Asian inhabitants of the United Kingdom than in the white population. ${ }^{1-4}$ However, longitudinal data comparing the pattern and extent of cardiovascular morbidity and mortality among hypertensive subjects belonging to these different racial groups in the United Kingdom are not available. Furthermore, no previous studies have evaluated racial differences in cardiovascular morbidity and mortality in relation to ambulatory blood pressure. Although cross sectional studies have generally shown ambulatory blood pressure to be more closely correlated with target organ damage than clinic blood pressure measurements, ${ }^{5}$ longitudinal studies comparing the prognostic significance of ambulatory versus clinic blood pressure measurements in essential hypertension are limited. ${ }^{67}$

A demographic study of 24 hour intraarterial ambulatory blood pressure monitoring in a hypertensive population of white, south Asian, and Afro-Caribbean subjects from our institution has previously been published. ${ }^{8}$ The aim of the present study was to compare the racial differences in cardiovascular morbidity and mortality in this hypertensive cohort in relation to baseline demographic characteristics and clinic and ambulatory blood pressure variables.

\section{Methods}

During the period 1 January 1979 to 1 January 1993, 723 patients were subjected to 24 hour intra-arterial ambulatory blood pressure monitoring at our institution. All patients were originally referred for the management of hypertension, based on a persistently raised clinic blood pressure. At the hospital visits, blood pressure was measured by a nurse or technician by the conventional auscultatory technique after 5-10 minutes of semisupine rest. The point of disappearance of auscultatory sounds was taken as the diastolic blood pressure. This was followed by a full medical history and physical examination by a physician.

Secondary causes of hypertension were excluded, as far as possible, by measurement of serum urea, creatinine, and electrolytes, urinary catecholamines, chest $x$ ray, and more recently intravenous renal digital subtraction angiography. Baseline clinic blood pressure was taken as the mean of two or more untreated readings at separate clinic visits, in the four weeks before or after the intra-arterial study. Those in whom clinic systolic blood pressure was $\geqslant 140 \mathrm{~mm} \mathrm{Hg}$ or diastolic blood pressure $\geqslant 90 \mathrm{~mm} \mathrm{Hg}$ were requested to undergo 24 hour intra-arterial ambulatory blood pressure monitoring within two months thereafter. Antihypertensive drug treatment had either not 
been started or had been withdrawn in the eight weeks preceding intra-arterial blood pressure monitoring.

The method of 24 hour intra-arterial ambulatory blood pressure monitoring was approved by the Harrow Health Authority ethics committee, and patients were required to give written informed consent prior to the procedure. General practitioners were informed of the results of the test and antihypertensive treatment was begun if considered appropriate on clinical grounds. Subsequent assessment of blood pressure control and treatment was largely left to the individual general practitioners or hospital physicians and was based on clinic blood pressure measurement.

INTRA-ARTERIAL BLOOD PRESSURE MONITORING The technique of intra-arterial blood pressure recording used in this laboratory has been well documented, ${ }^{9}$ as has the method of analysis. ${ }^{10}$ Blood pressure was recorded from a fine brachial artery cannula using a specially designed transducer/perfusion unit and an Oxford Medilog mark I tape recorder (Oxford, UK). The equipment was designed so that patients were fully ambulant and able to carry out their normal daily activities. The 24 hour tape recordings were analysed on a custom built hybrid computer, using a program which calculated mean hourly blood pressure. Mean systolic and diastolic intra-arterial blood pressure were calculated by averaging the 24 hourly systolic and diastolic readings. The daytime period was defined as the time interval between $6 \mathrm{am}$ and $10 \mathrm{pm}$ and the night time period as that between $10 \mathrm{pm}$ and 6 am. ${ }^{11}$ The nocturnal falls in systolic and diastolic blood pressure were calculated by subtracting respective night time mean from daytime mean blood pressure readings.

FOLLOW UP EVALUATION

Ethical approval for the most recent follow up, performed during an 18 months period from 1994 to 1996, was gained from the hospital ethics committee before contacting patients or their family practitioners. The dates and certified causes of death were obtained from the NHS central register, Southport, United Kingdom. The hospital records of all patients were scrutinised and survivors were invited to attend a follow up evaluation for documentation of events, clinic blood pressure measurement on current treatment, and fasting cholesterol concentration. General practitioners of the nonattenders were sent a questionnaire for details of these patients. As shown in the follow up scheme in fig 1, a minimum of two years of follow up data were available on 688 patients. These patients consisted of 528 white, 106 south Asian, and 54 Afro-Caribbean subjects. Of the south Asian subjects, 84 were Hindu (of whom 73 spoke Gujarati as their first language), 11 were Muslim, nine were AngloIndian, and two were Sikh.

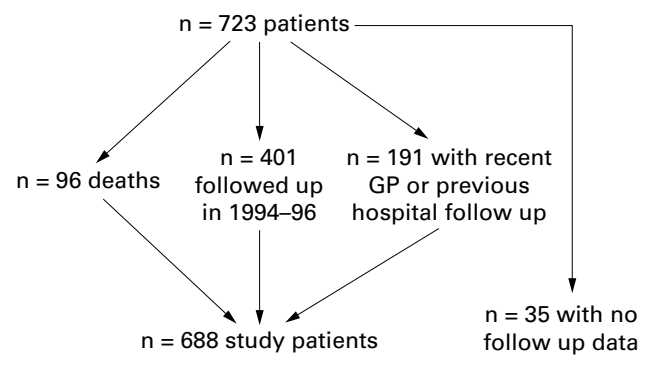

Figure 1 Follow up scheme for the 723 study patients.

EVALUATION OF DRUG TREATMENT AND BLOOD PRESSURE CONTROL

An assessment of antihypertensive treatment was made by subdividing the amount of drug treatment being taken by each individual into one of three categories: no treatment, monotherapy, or multiple therapy. An assessment of blood pressure control was made by comparing baseline clinic blood pressure with follow up clinic blood pressure. Complete information relating to drug treatment and blood pressure control was available on the 401 patients who attended follow up in 1994-96 (fig 1). In the group of non-attenders, data on drug treatment and follow up clinic blood pressure were retrieved either from departmental records of previous visits, from hospital notes from the medical records department, or from information obtained from the general practitioner. The follow up clinic blood pressures documented were the most current available or those taken before an event.

\section{CLASSIFICATION OF EVENTS}

Documented events were divided into noncardiovascular deaths, coronary events, cerebrovascular events, and peripheral vascular deaths. Coronary events consisted of coronary death (myocardial infarction or ischaemia, ventricular fibrillation, and cardiac failure), nonfatal myocardial infarction, coronary artery bypass graft surgery, and percutaneous transluminal coronary angioplasty. Cerebrovascular events included fatal and non-fatal stroke.

\section{STATISTICAL ANALYSIS}

The study population was divided into whites, south Asians, and Afro-Caribbeans to compare demographic, blood pressure, and events data in these racial groups. Continuous variables were expressed as mean (SD) and categorical variables as the percentage of patients so affected. A one way analysis of variance was performed to compare racial differences in age, body mass index, serum cholesterol, follow up period, clinic blood pressure, and ambulatory blood pressure variables. The Bonferroni multiple comparison procedure was used to compare the means of each continuous variable for each pair of racial groups. Fisher's exact test was performed to assess differences in sex distribution and smoking status. Finally, Cox's survival analysis was performed to determine the independent predictors of time to a first event with respective hazard ratios, 95\% confidence intervals and $\mathrm{p}$ values. A $\mathrm{p}$ value $<0.05$ was considered significant. 
Table 1 Comparison of baseline demographic variables among white, south Asian, and Afro-Caribbean subjects

\begin{tabular}{lllll}
\hline & $\begin{array}{l}\text { White } \\
(n=528)\end{array}$ & $\begin{array}{l}\text { Asian } \\
(n=106)\end{array}$ & $\begin{array}{l}\text { Afro-Caribbean } \\
(n=54)\end{array}$ & $p$ Value \\
\hline $\begin{array}{l}\text { Age (years) } \\
\text { Sex }\end{array}$ & $52.2(10.9)$ & $46.3(9.0)$ & $46.8(9.1)$ & $<0.001$ \\
$\quad$ Male & $327(62 \%)$ & $83(79 \%)$ & $29(54 \%)$ & \\
$\quad$ Female & $202(38 \%)$ & $22(21 \%)$ & $25(46 \%)$ & 0.001 \\
Smoking & $110(21 \%)$ & $21(20 \%)$ & $8(15 \%)$ & \\
$\quad$ Yes & $429(79 \%)$ & $84(80 \%)$ & $46(85 \%)$ & $\mathrm{NS}$ \\
$\quad$ No & $26.9(4.4)$ & $25.6(3.6)$ & $27.3(3.8)$ & 0.01 \\
BMI (kg/m $\left.{ }^{2}\right)$ & $6.0(1.2)$ & $5.6(1.1)$ & $5.5(1.0)$ & $<0.001$ \\
Cholesterol (mmol/1) & $5 \%$ & $17 \%$ & $15 \%$ & $<0.001$ \\
Diabetes mellitus & $7 \%$ & $7 \%$ & $7 \%$ & $\mathrm{NS}$ \\
Previous CV disease & $9.3(4.3)$ & $7.9(4.2)$ & $10.3(3.8)$ & 0.001 \\
Follow up (years) & & & & \\
\hline
\end{tabular}

Values are mean (SD) or $\mathrm{n}(\%)$.

$\mathrm{BMI}$, body mass index; CV, cardiovascular disease.

Table 2 Comparison of baseline clinic blood presure and ambulatory blood pressure variables among white, south Asian, and Afro-Caribbean subjects

\begin{tabular}{lllll}
\hline & $\begin{array}{l}\text { White } \\
(n=528)\end{array}$ & $\begin{array}{l}\text { Asian } \\
(n=106)\end{array}$ & $\begin{array}{l}\text { Afro-Caribbean } \\
(n=54)\end{array}$ & $p$ Value \\
\hline $\begin{array}{l}\text { Systolic blood pressure } \\
\text { Clinic systolic }\end{array}$ & $173.5(23.2)$ & $161.3(19.9)$ & $173.4(25.7)$ & $<0.001$ \\
24 hour systolic & $156.5(21.9)$ & $154.1(19.5)$ & $162.2(23.5)$ & NS \\
$\begin{array}{l}\text { Daytime systolic } \\
\text { Night time systolic }\end{array}$ & $163.8(21.9)$ & $162.0(19.8)$ & $169.1(23.9)$ & NS \\
Nocturnal fall in systolic & $141.4(24.3)$ & $137.3(21.1)$ & $147.3(24.6)$ & 0.04 \\
Diastolic blood pressure & $22.4(11.9)$ & $24.8(11.4)$ & $21.8(11.6)$ & NS \\
Clinic diastolic & $103.0(12.9)$ & $101.3(12.5)$ & $106.1(13.0)$ & NS \\
24 hour diastolic & $89.9(13.2)$ & $92.8(13.1)$ & $95.2(12.7)$ & 0.004 \\
Daytime diastolic & $95.0(13.3)$ & $98.1(13.4)$ & $100.0(12.9)$ & 0.006 \\
Night time diastolic & $79.0(14.9)$ & $81.4(14.0)$ & $84.8(14.2)$ & 0.01 \\
Nocturnal fall in diastolic & $16.0(8.5)$ & $16.8(8.0)$ & $15.2(8.5)$ & NS \\
& & & &
\end{tabular}

Values are mean (SD).
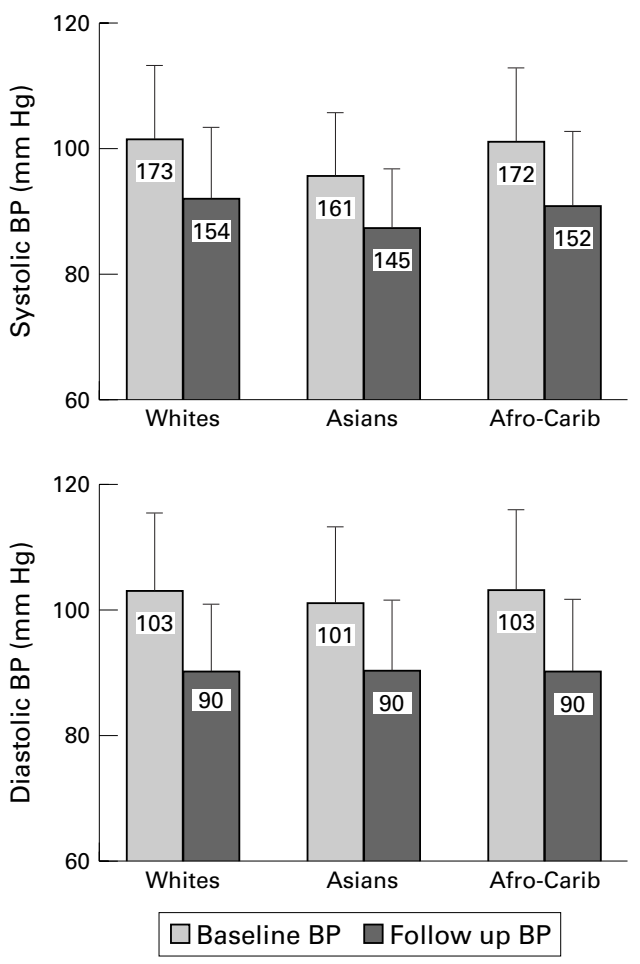

Figure 2 Baseline and follow up clinic blood pressures in white, south Asian, and Afro-Caribbean subjects.

clinic blood pressures in the 499 white, 103 south Asian, and 53 Afro-Caribbean subjects with complete clinic blood pressure data. South Asians had the lowest baseline and follow up clinic blood pressures of the three groups. The reductions in clinic systolic and diastolic blood pressure were $11 \%$ and $13 \%$ in whites, $10 \%$ and $11 \%$ in south Asians, and $11 \%$ and $13 \%$ in Afro-Caribbeans, respectively (NS). Complete information on drug treatment at follow up was available on 461 whites, 92 South Asians, and 51 Afro-Caribbean subjects. Eighty eight per cent of whites, $92 \%$ of south Asians, and all the Afro-Caribbean subjects were receiving antihypertensive treatment. Of these treated patients, $53 \%$ of whites, $41 \%$ of south Asians, and $53 \%$ of AfroCaribbeans were taking multiple treatment. The lower proportion of south Asians on multiple treatment may reflect the fact that average follow up clinic blood pressures were the lowest in this group.

Afro-Caribbeans had the highest and south Asians the lowest 24 hour mean, daytime mean, and night time mean ambulatory systolic blood pressures (table 2). Although these differences failed to reach statistical significance, Afro-Caribbeans had significantly higher 24 hour mean, daytime mean, and night time mean ambulatory diastolic blood pressure compared with whites, south Asians assuming an intermediate position. The nocturnal falls in blood pressure in the three groups were similar.

DRUG TREATMENT AND BLOOD PRESSURE

CONTROL

Blood pressure control was evaluated by determining the differences in baseline and follow up clinic blood pressure in the three groups. Figure 2 illustrates the baseline and follow up
EVENT RATES

A total of 157 first events was recorded, consisting of 32 non-cardiovascular deaths, 27 coronary deaths, 10 cerebrovascular deaths, four peripheral vascular deaths, 46 non-fatal myocardial infarctions, 20 non-fatal strokes, and 18 coronary revascularisations. South Asians had the highest all cause event rate of 3.46 events/100 patient-years compared with 2.50 and 0.90 events $/ 100$ patient-years for whites and Afro-Caribbeans, respectively $(\mathrm{p}=0.01)$. The high event rate in south Asians was because of an excess of coronary events (fig 3). Of the 29 first events documented in south Asians, 24 were coronary in nature; south Asians had more than twice as many coronary 


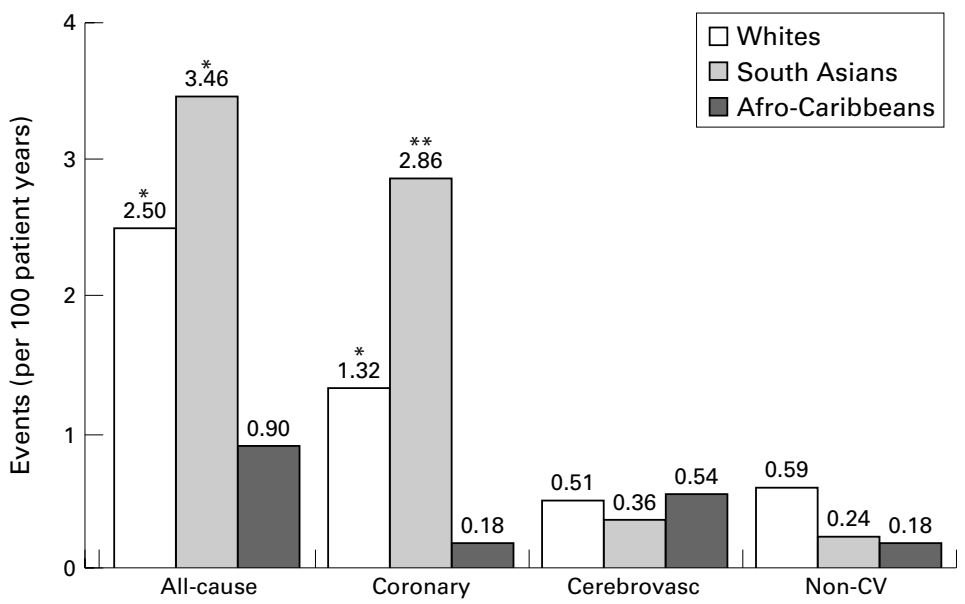

Figure 3 Incidence of non-cardiovascular, coronary, and cerebrovascular events in white, south Asian, and Afro-Caribbean subjects. ${ }^{\star} p<0.01 v$ Afro-Caribbeans; ${ }^{\star *} p<0.01 v$ whites and Afro-Caribbeans.

Table 3 Independent predictors of time to a first event with hazard ratios, 95\% confidence intervals, and $p$ values

\begin{tabular}{llll}
\hline & Hazard ratio & $95 \%$ CI & p Value \\
\hline Age (/year increase) & 1.06 & 1.04 to 1.08 & $<0.001$ \\
Sex (male:female) & 2.39 & 1.56 to 3.66 & 0.002 \\
Race (south Asian:white) & 1.79 & 1.16 to 3.66 & 0.008 \\
Diabetes (yes:no) & 1.65 & 1.00 to 2.73 & 0.05 \\
Previous CV disease (yes:no) & 2.85 & 1.85 to 4.38 & $<0.001$ \\
24 h systolic BP (/10 mm Hg increase) & 1.14 & 1.06 to 1.23 & 0.006 \\
\hline
\end{tabular}

$\mathrm{BP}$, blood pressure; $\mathrm{CI}$, confidence interval; $\mathrm{CV}$, cardiovascular disease.

events as whites (2.86 $v 1.32$ events/100 patient-years, respectively; $\mathrm{p}=0.002)$. Only five Afro-Caribbean subjects experienced an event; these were three cerebrovascular events, one coronary event, and one noncardiovascular death. Each of the four peripheral vascular deaths occurred in white patients.

\section{MULTIVARIATE ANALYSIS}

Table 3 summarises the results of Cox proportional hazards regression analysis with respective hazard ratios, 95\% confidence intervals, and $p$ values. Age $(p<0.001)$, male sex $(\mathrm{p}<0.001)$, south Asian origin (south Asians $v$ whites: $p=0.008$ ), diabetes mellitus $(p=0.05)$, previous history of cardiovascular disease $(p<0.001)$, and 24 hour ambulatory systolic blood pressure $(\mathrm{p}=0.006)$ were independent predictors of time to a first event.

\section{Discussion}

This study provides the first longitudinal prognostic data comparing cardiovascular morbidity and mortality in hypertensive white, south Asian and Afro-Caribbean subjects. Despite younger age, lower serum cholesterol, lower body mass index, and a trend towards lower systolic blood pressure at baseline, south Asians had a higher all cause event rate than whites. The only traditionally established risk factor more prevalent among south Asians was diabetes mellitus, ${ }^{12} 13$ but even after correction for this, south Asians had nearly twice the risk of a morbid event compared with their white counterparts (table 3). Although cerebrovascular disease might have been expected to contribute to the high event rate in this hypertensive population, the major cause of the excess morbidity and mortality in south Asians was the susceptibility to coronary heart disease. Epidemiological data derived from the general population of the United Kingdom ${ }^{14}$ and other countries ${ }^{15}$ confirm a uniformly high incidence and mortality from coronary heart disease in south Asians. The low event rate in the Afro-Caribbean group in our study may relate to their relatively young age, lower proportion of male subjects, the omission of heart failure admissions and renal failure as primary endpoints, and the known low incidence of coronary heart disease in this racial group.

The considerably increased risk of coronary events in south Asian hypertensive subjects highlights the need for intensive risk modification strategies in this racial group in particular. The reasons for the excess coronary risk are multifactorial and include not only the role of conventional cardiovascular risk factors but also other incompletely understood genetic and metabolic mechanisms, not evaluated in our study. It has been suggested by McKeigue et al that even in non-diabetic south Asians, the syndrome of insulin resistance may at least in part be responsible for the adverse cardiovascular outcome in these subjects. ${ }^{16}$ Many reports have confirmed that south Asians have a predisposition to insulin resistance characterised by central obesity, hyperinsulinaemia, hypertriglyceridaemia, and low HDL cholesterol, ${ }^{16-18}$ and these metabolic abnormalities may interact to promote atherogenesis. Other risk factors for coronary heart disease including serum levels of lipoprotein (a) and homocysteine have also been shown to be elevated in south Asians. ${ }^{19-21}$

In comparison with the wealth of epidemiological and intervention data on clinic based blood pressure measurements, there are few longitudinal data evaluating the clinical and prognostic implications of ambulatory blood pressure. Our study reports the longest period of follow up data to evaluate the predictive value of ambulatory versus clinic blood pressure measurement. The best regression model for the prediction of time to a first event showed that baseline 24 hour ambulatory systolic blood pressure was an independent predictor of all cause morbidity and mortality, whereas clinic blood pressure failed to provide independent prognostic information. This independent relation between baseline ambulatory blood pressure and subsequent morbidity and mortality was present despite the administration of interim drug treatment in the vast majority of patients. Since antihypertensive treatment could only have served to weaken this relation, it is possible that the true association may have been even stronger, suggesting that an assessment of 24 hour blood pressure load, particularly systolic blood pressure, may be a superior method of identifying high risk patients. A recent prospective longitudinal study showed that regression of left ventricular hypertrophy was more closely predicted by treatment induced changes in ambulatory blood pressure than in clinic blood pressure. ${ }^{22}$ Whether treatment based on ambulatory rather than clinic blood pressure has a 
greater impact on the prevention of overt cardiovascular disease remains to be evaluated.

LIMITATIONS

As the data on drug treatment and blood pressure control were obtained in a retrospective manner, interpretation of their findings should be made with caution. In view of the variety of sources used to obtain follow up clinic blood pressures, the environmental conditions, methodological approach, equipment used, and type of personnel performing the measurements may have been different from the baseline measurements. Moreover, the time interval between baseline and follow up clinic blood pressure measurements was not formalised and may have varied from individual to individual. Nevertheless, these limitations applied equally to each racial group and despite these limitations the magnitude of the observed reductions in blood pressure were feasible and similar.

We gratefully acknowledge the advice and support of the late Dr We gratefully acknowledge the advice and support of the late Dr E B Raftery. The technical support of Chris Kinsey and Ann
Banfield and the statistical services of Caroline Dore are also very much appreciated.

1 Cappucio FP, Cook DG, Atkinson RW, et al. Prevalence detection and management of cardiovascular risk factors in different ethnic

2 Raleigh VS. Diabetes and hypertension in Britain's ethnic minorities: implications for the future of renal services. BMF 1997;314:209-13

3 Meade TW, Brosovic M, Chakraborti R, et al. Ethnic group comparisons of variables associated with ischaemic hear disease. Br Heart $\mathcal{F}$ 1978;40:789-95.

4 Venkata C, Ram S. Hypertension and other cardiac risk factors among Asian Indians. Am f Hypertens 1995;8:124-7S

5 Clement DL, De Buyzere M, Duprez D. Prognostic value of ambulatory blood pressure monitoring. F Hypertens 1994 12:857-64

6 Perloff D, Sokolow M, Cowan R. The prognostic value of ambulatory blood pressures. $\mathcal{F} A M A$ 1983;249:2792-8.
7 Verdecchia P, Porcellati C, Schillaci C, et al. Ambulatory blood pressure: an independent predictor of prognosis in essential hypertension. Hypertension 1994;24:793-801.

8 Acharya DU, Heber ME, Dore CJ, et al. Ambulatory intraarterial blood pressure in essential hypertension: effects of age, sex race and body mass - the Northwick Park Hospital Database study. Am f Hypertens 1996;9:943-52.

9 Millar-Craig MW, Bishop CN, Raftery EB. Circadian variation of blood pressure. Lancet 1978;i:795-7.

10 Cashman PM, Stott FD, Millar-Craig MW. Hybrid system for fast data reduction of long-term blood pressure recordings. Med Biol Eng Comput 1979;17:629-35.

11 Verdecchia P, Schillaci G, Guerrieri M, et al. Circadian blood pressure changes and left ventricular hypertrophy in essential hypertension. Circulation 1990;81:528-36.

12 Mather HM, Keen H. The Southall diabetes survey: prevalence of known diabetes in Asians and Europeans. BMF 1985;291:1081-4.

13 Omar MAK, Seedat MA, Dyer RB, et al. The prevalence of diabetes mellitus in a large group of South African Indians. South Afr Med f 1985;67:924-6.

14 Balarajan R. Ethnic differences in mortality from ischaemic heart disease and cerebrovascular disease in England and Wales. BM7 1991;302:560-4.

15 McKeigue PM, Miller GJ, Marmot MG. Coronary heart disease in South Asians overseas - a review. 7 Clin Epidemiol 1989;42:597-609.

16 McKeigue PM, Shah G, Marmot MG. Relation of central obesity and insulin resistance with high diabetes prevalence and cardiovascular risk in South Asians. Lancet 1991;337: $382-6$.

17 McKeigue PM, Marmot MG, Syndercombe Court YD, et al. Diabetes, hyperinsulinaemia and coronary risk factors in Bangladeshis in East London. Br Heart f 1988;60:3906.

18 Knight TM, Smith Z, Whittles A, et al. Insulin resistance, diabetes and risk markers for ischaemic heart disease in Asian men and non-Asian men in Bradford. Br Heart $\mathcal{f}$ 1992;67:343-50.

19 Bhatnagar D, Anand IS, Durrington PN, et al. Coronary risk factors in people from the Indian subcontinent living in West London and their siblings in India. Lancet 1995;345: 405-9.

20 Anand SS, Enas EA, Pogue J, et al. Elevated lipoprotein(a) levels in South Asians in North America. Metabolism 1998; 47:182-4.

21 Chambers J, Kooner JS. Homocysteine levels are elevated in UK Indian Asians compared to British white men. Heart 1998;79(suppl 1):P59.

22 Mancia G, Zanchetti A, Agabitei-Rosei E, et al. Ambulatory blood pressure is superior to clinic blood pressure in predicting treatment-induced regression of left ventricular hypertrophy. Circulation 1997;95:1464-70. 